\title{
Sketch-Based 3D Model Retrieval using Diffusion Tensor Fields of Suggestive Contours
}

\author{
Sang Min Yoon \\ GRIS, TU Darmstadt \\ Fraunhoferstr. 5 \\ 64283, Darmstadt, Germany \\ sangmin.yoon@ \\ gris.tu-darmstadt.de
}

\author{
Maximilian Scherer \\ GRIS, TU Darmstadt \\ Fraunhoferstr. 5 \\ 64283, Darmstadt, Germany \\ maximilian.scherer@ \\ gris.tu-darmstadt.de
}

\author{
Arjan Kuijper \\ Fraunhofer IGD \\ Fraunhoferstr. 5 \\ 64283, Darmstadt, Germany \\ arjan.kuijper@igd.fraunhofer.de
}

\author{
Tobias Schreck \\ GRIS, TU Darmstadt \\ Fraunhoferstr. 5 \\ 64283, Darmstadt, Germany \\ tobias.schreck@ \\ gris.tu-darmstadt.de
}

\begin{abstract}
The number of available 3D models in various areas increases steadily. Effective methods to search for those 3D models by content, rather than textual annotations, are crucial. For this purpose, we propose a new approach for content based 3D model retrieval by hand-drawn sketch images. This approach to retrieve visually similar mesh models from a large database consists of three major steps: (1) suggestive contour renderings from different viewpoints to compare against the user drawn sketches; (2) descriptor computation by analyzing diffusion tensor fields of suggestive contour images, or the query sketch respectively; (3) similarity measurement to retrieve the models and the most probable view-point from which a model was sketched. Our proposed sketch based 3D model retrieval system is very robust against variations of shape, pose or partial occlusion of the user drawn sketches. Experimental results are presented and indicate the effectiveness of our approach for sketch-based 3D model retrieval.
\end{abstract}

\section{Categories and Subject Descriptors}

I.3.8 [Computing methodologies]: Computer GraphicsApplications

\section{General Terms}

Algorithms

\section{Keywords}

3D model retrieval, suggestive contours, diffusion tensor field

\section{INTRODUCTION}

With the rapid increase in the number of available 3D models, the ability to accurately and effectively search for $3 \mathrm{D}$ model is crucial for many applications such as industrial design, engineering, and manufacturing area.

The need to search for 3D models using their content, rather than textual annotations, has led to the development of several approaches to compute the similarity between two 3D models in recent years [1] by using several algorithms like shape histogram [2], shape distribution [3], moment [4], light field [5], spherical harmonics [6]. Following such an approach, users can search for 3D models by supplying an example query object. Depending on the task, 3D model retrieval using query-by-example is not the most intuitive approach. It assumes that users already have a well defined $3 \mathrm{D}$ query model, which is similar to what they are searching for.

Another approach to search a database of 3D models relies on two dimensional, user-drawn sketches. Among numerous interaction methodologies, humans can sketch important features of objects they are interested in very quickly. Although a sketch is composed of only a few lines, it is a coarse but detailed picture including key features. It is particularly suited for 3D model retrieval, as the human visual system perceives 3D models as two dimensional projections. To compute the similarity between a 3D model and a usersketch image, the model is usually projected and rendered from several viewpoints and the best-matching similarity between the sketch image and the projected images are computed. Several techniques used in content-based image retrieval [7-9] to search and rank a set of similar images in a large database, can also by used for sketch-based 3D model retrieval.

In this paper we propose a novel approach for sketchbased model retrieval by computing view-based descriptors using suggestive contours [10]. This technique from nonphotorealistic rendering resembles hand-drawn sketches of three dimensional objects very closely. We project the suggestive contours of each 3D model from several predefined viewpoints and compute a feature vector based on the orientation of these contours. The same way a feature vector 


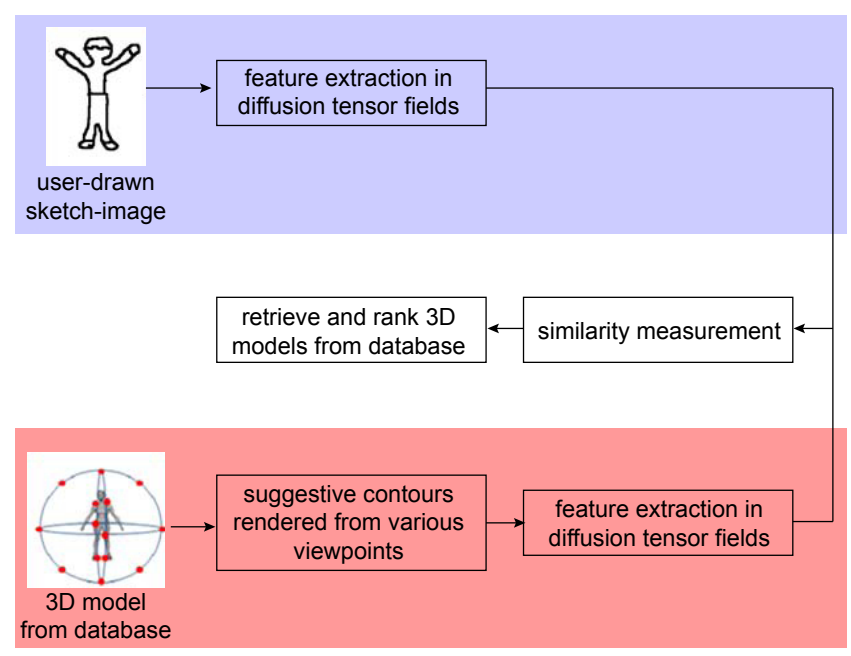

Figure 1: Flowchart of our sketch-based 3D model retrieval approach.

can be computed from a user-drawn sketch. By comparing such feature vectors we can rank the $3 \mathrm{D}$ models according to their similarity to the user-sketch. Since we projected each $3 \mathrm{D}$ model from several viewpoints, we can also retrieve the (approximate) most-likely view-point the user had in mind when drawing the sketch.

In Figure 1 a flowchart shows, how we extract meaningful features from 3D models as well as query images. Our proposed system is composed of three steps: extract the suggestive contours from different viewpoints; feature analysis in the topological space of diffusion tensor fields; similarity measure using histogram of orientation.

The remaining part of this paper is organized as follows. In Section 2 we briefly survey related work in the area of $3 \mathrm{D}$ model retrieval and diffusion tensor fields. In Section 3 we explain in detail, how we extract features from 3D models using suggestive contour images and measure similarity using histograms of orientation based on the properties of diffusion tensor fields. In Section 4 we evaluate and present experimental results using query images sketched by several users. We further discuss those results and implications in Section 5 .

\section{RELATED WORK}

User drawn sketch based 3D model retrieval is closely related to various areas in computer vision and computer graphics. In this section, we briefly survey previous work of $3 \mathrm{D}$ model retrieval and the use of diffusion tensor fields.

\subsection{D Model Retrieval}

There are numerous approaches in 3D model retrieval to compute the similarity between two objects. A good overview can be found in [1]. Approaches can be distinguished by supporting global or partial model similarity. Global methods determine the overall similarity of the entire shape, while partial methods analyze for local similarities. One prominent approach for global similarity is the computation of a high-dimensional feature vector, that tries to capture discriminative properties of the model, while being robust against minor changes of the model's shape.

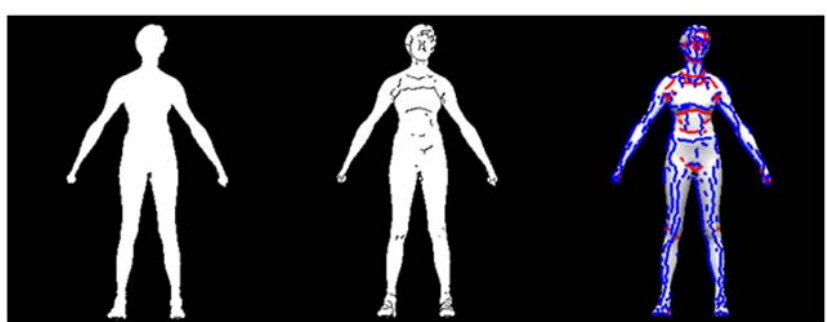

Figure 2: Comparison of contour, suggestive contour, and ridge and valleys. Suggestive contours provide more detailed information of 3d models than boundary contours and ridges and valleys. In particular, the suggestive contour is very suited when 3D models have a smooth shape.

Our approach also computes a feature vector to describe the global shape of a model. Since we aim for sketch-based $3 \mathrm{D}$ model retrieval we compute view-based features, meaning we project and render 3D models as images from several view-points to match them against a user-drawn sketch image. Other such view-based approaches are presented in [11], which was only used for query-by-example, and [12], which was also successfully used for sketch-based retrieval.

Methods from 2D shape analysis and content-based image retrieval also become applicable to compute a feature vector of each view-image. Respective surveys giving an overview of prominent methods are [13] and [14].

Since we cannot assume that each user-drawn sketch is a closed contour, established contour-based shape descriptors like Curvature Scale Space [15] are not applicable. Regionbased shape descriptors like Zernike Moments are applicable on the other hand, and were successfully used for the recognition of sketched symbols in [16]. From the features of the suggestive contour, we heuristically encode magnitude and orientation properties of the diffusion tensor field as a histogram. This relates to the rather successful image descriptor Histograms of Oriented Gradients (HOG) [17] used primarily for detection and recognition tasks in computer vision.

Apart from computing the similarity between user-drawn images and view-point images by selecting a suitable descriptor, the challenge of projecting and rendering a 3D model in a meaningful way remains.

Previous approaches for sketch-based 3D model retrieval rely on projecting the silhouette (contour) of a model [18$22]$. Such a rendering does not correspond to the way most people naturally draw. Looking in the direction of nonphotorealistic rendering techniques [23], suggestive contours [10] were created with the goal to resemble artistic sketches of $3 \mathrm{D}$ objects as closely as possible. Accordingly we argue, that this rendering technique is particularly suited for sketch-based model retrieval.

One area where sketch-based model retrieval is successfully used, is model retrieval in CAD design for engineering. View-based projections resembling not artistic sketches but rather technical drawings are employed in this field $[24,25]$.

\subsection{Diffusion Tensor Fields}

Diffusion tensor fields (with a focus on symmetric, second order ones) are used in many medical, mechanical, and 


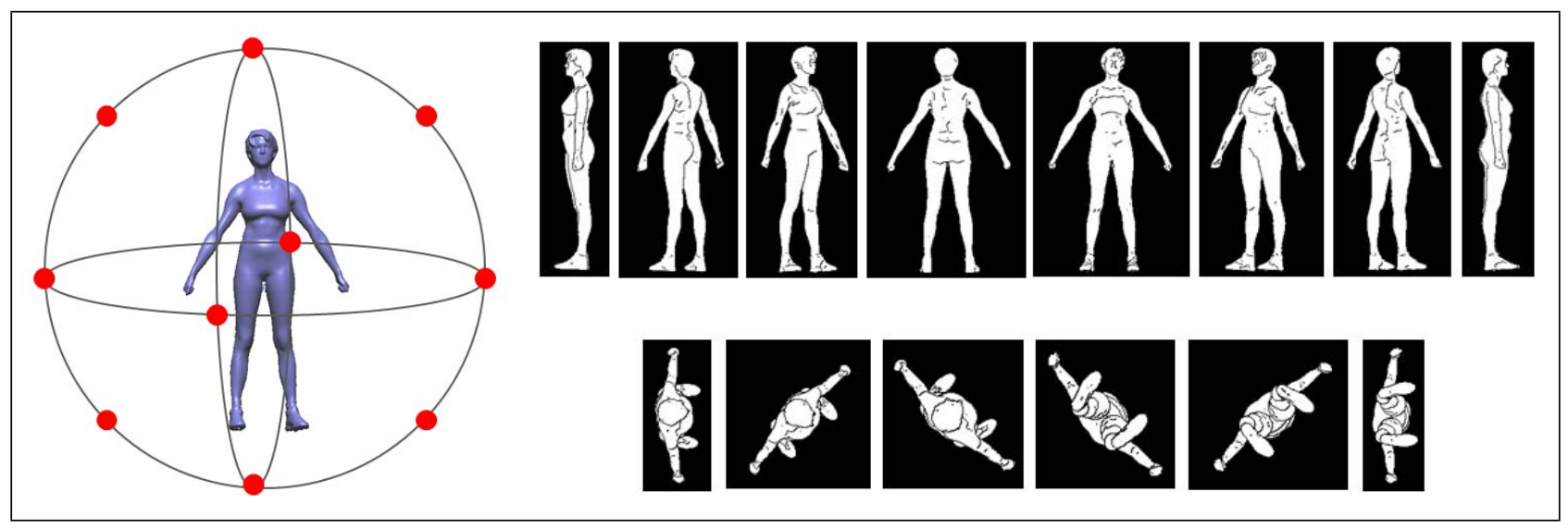

Figure 3: A 3D model and its suggestive contours from 14 different viewpoints. 6 orthographic and 8 isometric projection images are used to measure the similarity between a user drawn sketch and a 3D model.

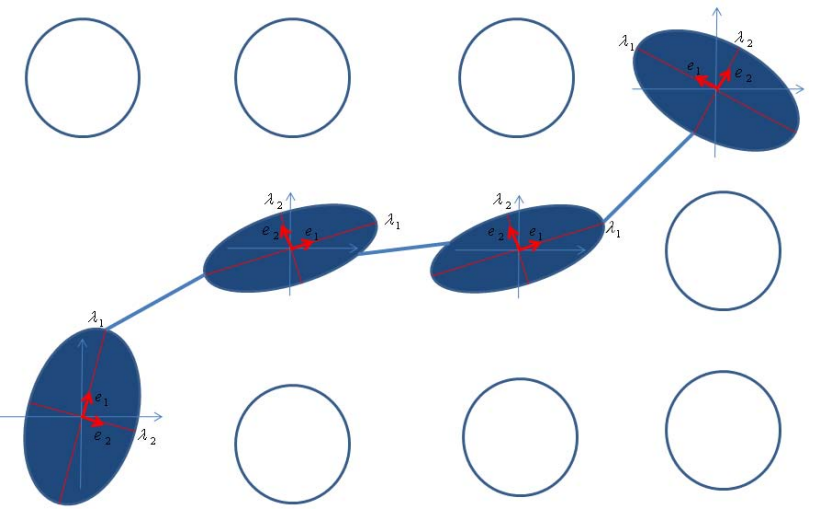

Figure 4: Ellipsoidal representation of eigenvalues and eigenvectors which come from the properties of diffusion tensor fields. Each pixel of the original image is represented as an ellipse. The ellipses painted in blue are actual elements of the suggestive contour.

physical applications such as fluid dynamics, meteorology, molecular dynamics, biology, astrophysics, mechanics, material science and earth science. [26] presented their work on diffusion tensor magnetic resonance imaging (DT-MRI). Using this new MRI modality, it was possible to qualify anisotropic properties of the imaged tissue by characterizing the water diffusion.

We convert binary images to their corresponding diffusion tensor fields. This enables us to analyze the orientation of each pixel, depending on its neighborhood.

\section{OUR APPROACH}

Our approach for 3D model retrieval from hand drawn sketch images evaluates similarity by comparing the query image to 14 projected views of the model. For each such image, we extract a histogram of orientation from the corresponding diffusion tensor field. The details for this approach are explained in the following subsections.

\subsection{Suggestive Contour Extraction from Dif- ferent Viewpoints}

In multimedia retrieval it is very important to extract descriptive features and measure similarity between objects as closely to the user's intentions as possible.

For sketch-based retrieval of 3D models, without restricting ourselves to a specific domain like architecture or mechanical engineering, we argue that visual similarity from a specific viewpoint is the most likely use case. Finding appropriate projection and rendering techniques, that resemble rough user sketches closely, is crucial. For example rendering the depth map of a 3D model is certainly very descriptive of the mesh itself, but it is not feasible to expect users to draw and shade a correct depth map.

The silhouette of a model on the other hand is sketched easily, but any view-dependent information is lost. Rendering all ridges and valleys certainly conveys $3 \mathrm{D}$ shape, but again the view-dependent nature, which hand-drawn sketches posses, is lost. Sketching skeletal features of a 3D model, especially for humanoid models, is very intuitive ("stick-men"), but again any information relating to the view or depth of the object itself is lost.

To overcome the drawbacks of previous approaches, we propose to extract Suggestive Contours [10] to construct descriptors from different viewpoints. A suggestive contour rendering closely resembles the way most people sketch three dimensional objects: The silhouette as well as major ridges and valleys of the model are outlined (see Figure 2). This is a very minimalistic way to convey three dimensional shape by a two dimensional image.

To be able to compare 3D models and user sketches, we render the suggestive contour of each model from 14 different, equally spaced viewpoints (see Figure 3). We use 6 orthographic projections and 8 isometric projections. We use these 14 viewpoints as a compromise between accuracy to the viewpoint the user had in mind when sketching the query, and processing time of the algorithm.

Once we rendered all 14 views of a 3D model we extract feature vectors from each of the views and the query sketch image itself. By computing the minimal distance between the feature vector of the query and each view image, we can rank all models from the database accordingly. In addition 


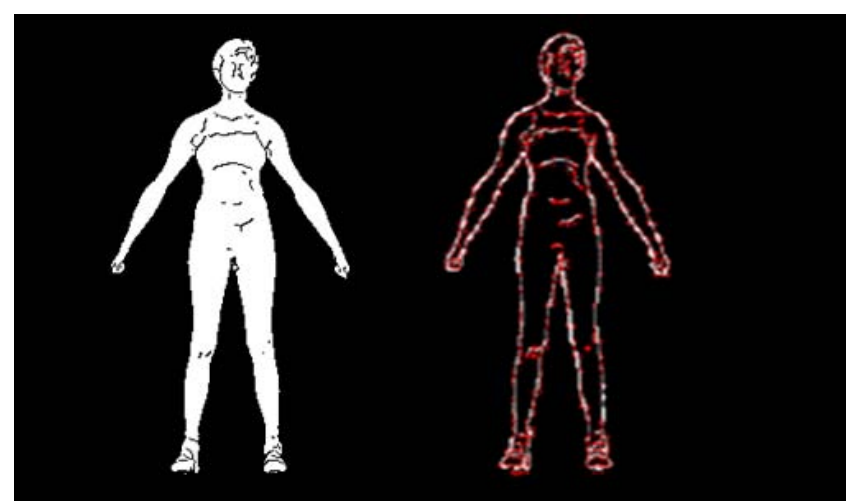

Figure 5: Ellipsoidal representation of suggestive contours

the closest view to the query image is also the approximate viewpoint, the user had in mind when sketching the query. Details on the feature vector extraction are presented in the next subsection.

\subsection{Feature Analysis using Diffusion Tensor Fields}

To extract a feature vector from each suggestive contour image and the query image itself, we analyze its properties in the space of diffusion tensor fields.

Diffusion tensor fields are originally introduced in the area of medical image processing to measure the diffusion of water in tissue [26]. If the tissue is fibrous, the water molecules diffuse more in directions along the fibers than perpendicular to them. Using this technique, it is possible to analyze the motion of deformable objects which have a high degree of freedom [27].

A tensor is a mathematical definition of a geometric or physical quantity whose analytic description consists of an array of numbers. This means that a tensor is an abstract object expressing some definite type of multi-linear concept. The tensor field - commonly defined as a topological representation of a 2D symmetric, second-order tensor field - is given by :

$$
T=\left(\begin{array}{ll}
T_{x x} & T_{x y} \\
T_{y x} & T_{y y}
\end{array}\right)
$$

where $T_{x y}=T_{y x}$. So this corresponds to a symmetric positive definite matrix.

This matrix can be reduced to principal axes by solving the characteristic equation

$$
(T-\lambda \cdot \mathbf{I}) \mathbf{e}=\mathbf{0},
$$

where $\mathbf{I}$ is the identity matrix, $\lambda$ are the eigenvalues of the tensor and $e$ are the normalized eigenvectors and the corresponding eigenvectors are orthogonal. In this case, the tensor in each pixel can be represented by an ellipsoid, where the main axis lengths are proportional to the eigenvalues $\lambda_{1,2}$ $\left(\lambda_{1}>\lambda_{2}\right)$.

Figure 4 shows the ellipsoidal representation of the suggestive contour elements which are painted in blue. Each pixel of the suggestive contours within a projected image is represented as a two-dimensional ellipsoid, whose direction and scale are determined by the corresponding eigenvalues and

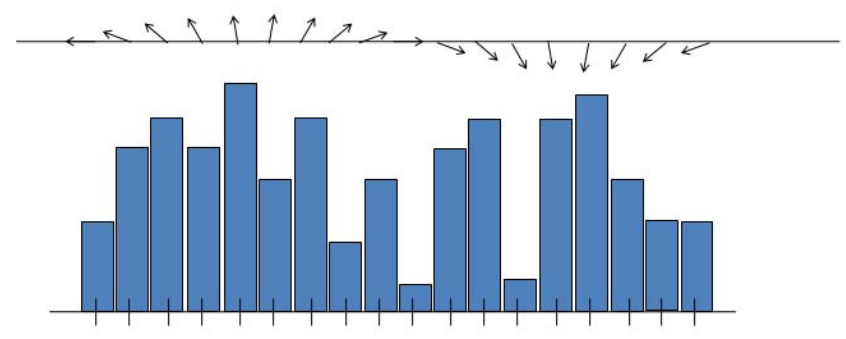

Figure 6: Histogram of Orientation of all pixels regarded in the corresponding diffusion tensor field.

eigenvectors from the diffusion tensor field. These properties of each ellipse are then organized into a histogram according to their orientation and their magnitude, which will be explained in the next subsection.

Figure 5 shows such an ellipsoidal representation of the suggestive contour of one of the projected views of a $3 \mathrm{D}$ model.

The extracted eigenvalues and eigenvectors which come from diffusion tensor fields are used to compute a feature vector of the suggestive contour views of the $3 \mathrm{D}$ model, or the user sketch respectively.

\subsection{Similarity Measure Based on Histogram of Orientation}

Given a pair of images, $I_{c}$ that is a query image, and $I_{s}$ that is a suggestive contour image from a $3 \mathrm{D}$ model, normalized to a fixed size, we define an aligned distance score that accounts for the normalized deformation between the two images. Using the properties of the ellipsoidal representation of each contour pixel, we compute histogram-based feature vectors $H_{c}$ and $H_{s}$ as follows:

1. We extract the magnitude $m(x, y)$ and orientation $\theta(x, y)$ of the ellipsoidal representation of each contour pixel. As aforementioned, the ellipses are defined by the eigenvalues and eigenvectors from the analysis of the suggestive contour in the topological space of diffusion tensor fields.

2. We quantize the orientation into $n$ orientation bins weighted by the corresponding magnitude $m(x, y)$. We quantize the orientation into 18 bins which are shown in Figure 6. The quantized orientation is extracted from the direction of main axis of the ellipsoidal representation of the suggestive contour. The main direction of the ellipsoidal model is determined by the eigenvector $e_{1}$.

3. The resulting feature vector of histogram of orientation, $H_{s}$ and $H_{c}$, are normalized into unit length vector by the sums of all entries

4. The similarity between the query image and one view image of a $3 \mathrm{D}$ model, $S\left(I_{c}, I_{s}\right)$ is then given by the following equation:

$$
S\left(I_{c}, I_{s}\right)=\frac{H_{c} \cdot H_{s}}{\left\|H_{c}\right\|\left\|H_{s}\right\|}
$$

Note that the value of $S\left(I_{c}, I_{s}\right)$ lies in the interval [0,1]. If the histograms $I_{c}$ and $I_{s}$ are identical then $S\left(I_{c}, I_{s}\right)=1$.

5. For user drawn sketch based $3 \mathrm{D}$ model retrieval, we projected the $3 \mathrm{D}$ model into 14 different viewpoints. The similarity score between a query image and a $3 \mathrm{D}$ model is 


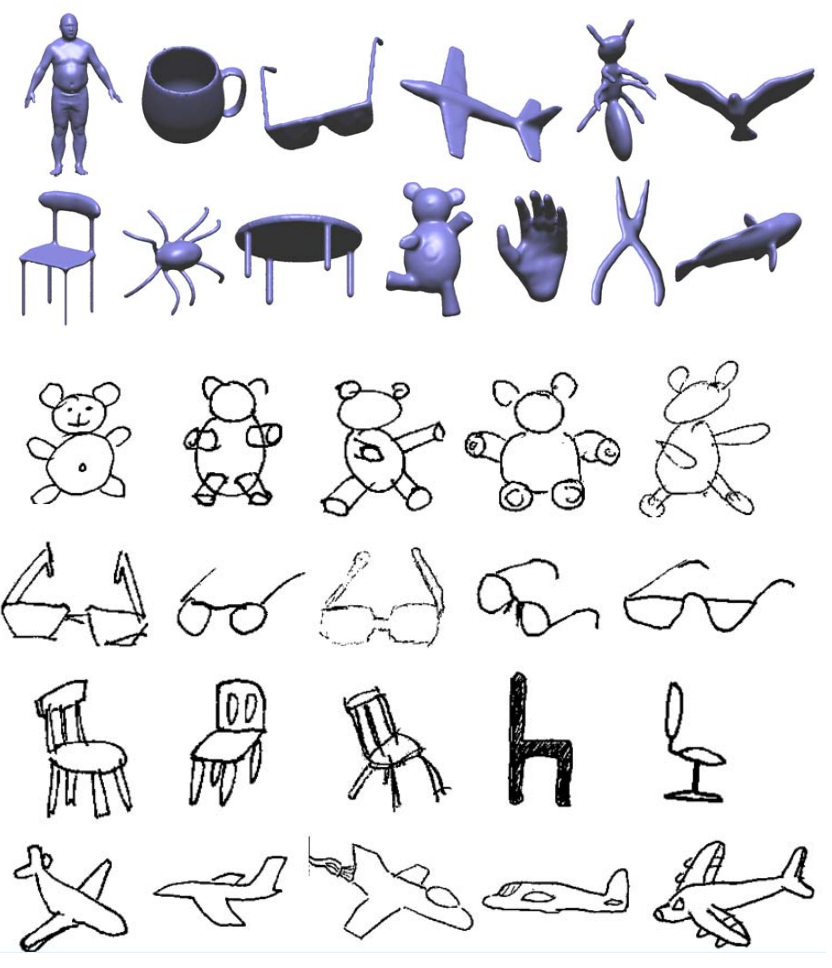

Figure 7: Representative 3D models and user drawn sketched query images which are used for our experiments.

determined by the maximum similarity $\max \left(S\left(I_{c}, I_{s}\right)\right)$, between the query image and one of the 14 view-descriptors.

The advantage of histogram of orientation in the space of diffusion tensor fields is that it is very robust against partial occlusion or translation of the user drawn sketched to the target 3D model.

\section{EXPERIMENTS}

In this section, we conduct several experiments to evaluate the effectiveness of our proposed method. This section is separated into 3 parts: experimental setup; retrieval results from various sketched query images by numerous users; comparison of our method against methods using different rendering techniques.

\subsection{Experimental setup}

We conducted several experiments to evaluate the retrieval performance of our approach. For our experiments, we used several 3D mesh models from the Princeton Shape Benchmark (http://segeval.cs.princeton.edu). We used 260 models from 13 classes, i.e. "human", "cup", "airplane", "ant", "chair", "sunglasses", etc.

Accordingly we rendered 260x14 suggestive contour images, which we used for our evaluation. We also collected 250 user drawn sketch images from numerous users. We used the 14 different viewpoints of a 3D model to retrieve the 3D models. In comparison, Funkhouser et al. used 13 orthographic viewpoints [18], Chen et al. used 10 shaded boundary images from 20 viewpoints [19], and Macrini et al. [20] used 128 projected images to 3D model retrieval.
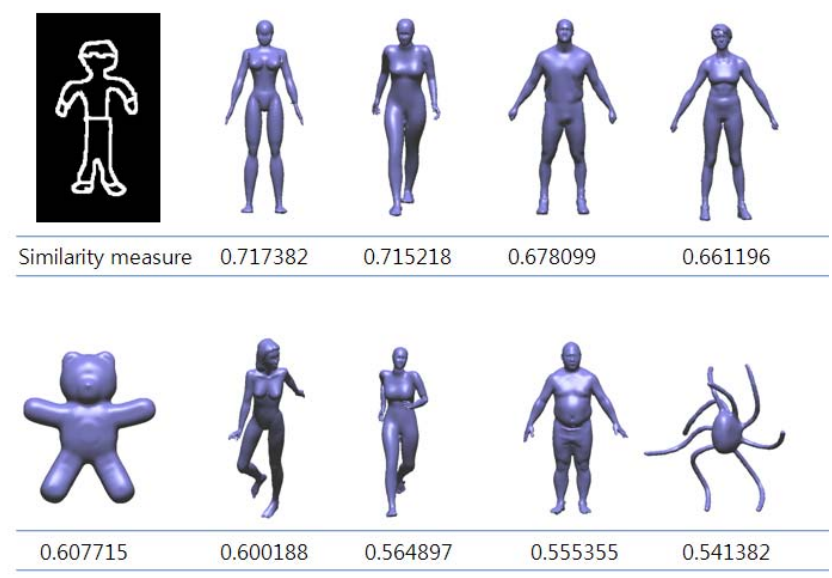

(a) The retrieved models and their respective similarity to an example sketch-query-image.

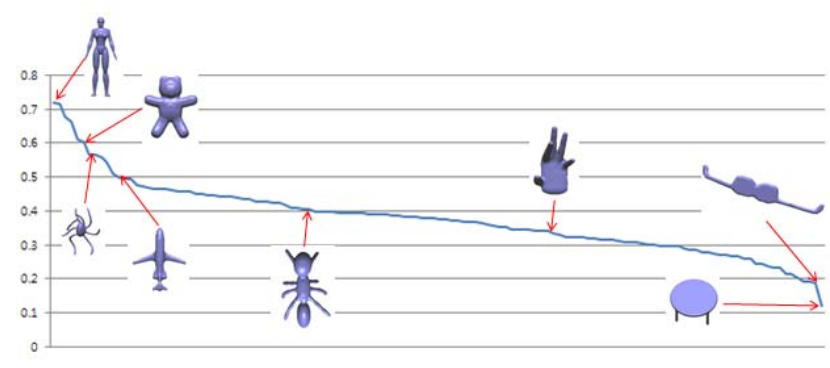

(b) The variation of the similarity measure of the retrieved 3D models to the query.

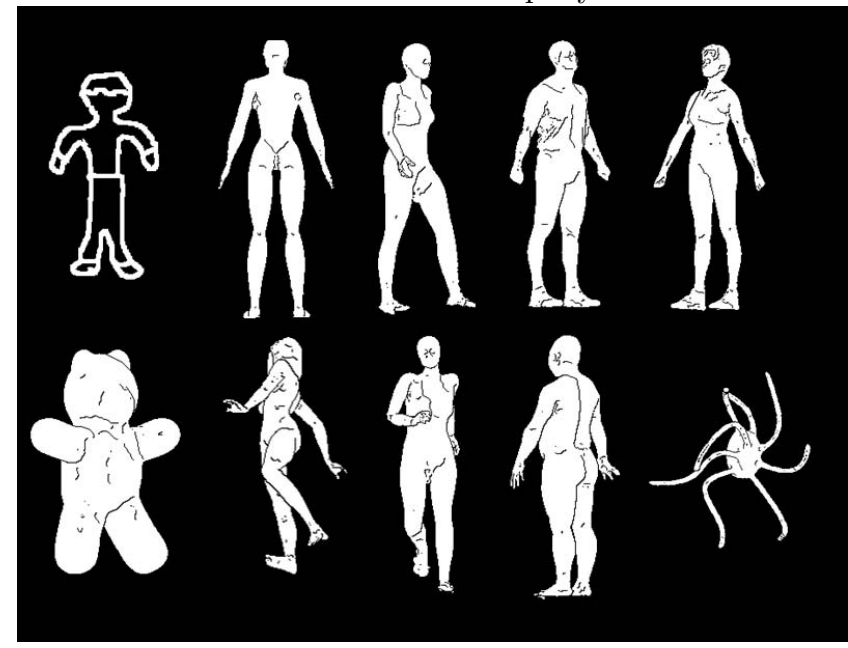

(c) The most similar viewpoints of the retrieved 3D models from a sketched query image.

Figure 8: 3D model retrieval and its viewpoints from an example sketched query image. 


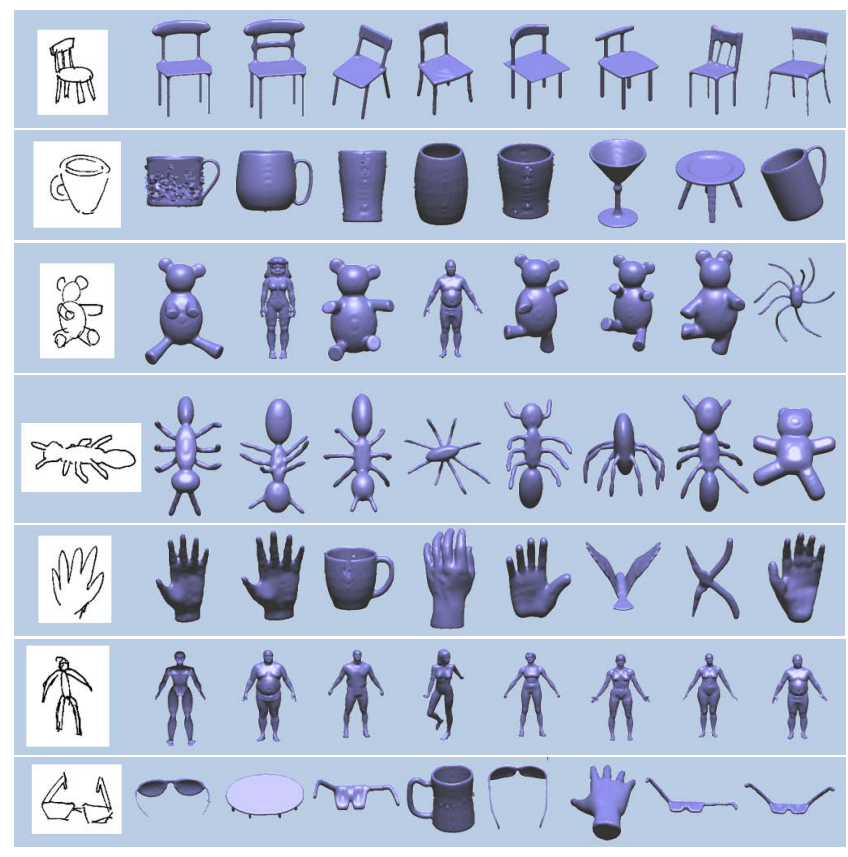

Figure 9: 3D model retrieval results from various sketched query images.

Figure 7(a) shows the represented 3D models and user drawn sketches which are used for our experiments. Figure 7(b) shows the sketched query images of "bear", "sunglasses", "chair", and "airplane" which are drawn by different users. Their query images are very different according to their interest of the 3D model. For example, the query images of chair have various viewpoints.

To measure the similarity, we first crop the user drawn sketched query image and normalize its size to 200x200 pixels and then extract its eigenvalues and eigenvectors of the corresponding diffusion tensor field.

\subsection{Evaluation of Sketch-Based 3D Model Re- trieval}

In this section, we show the results of the retrieved $3 \mathrm{D}$ models from various user drawn sketches.

We first analyze the retrieved 3D models by one sketched query image and variation of its similarity measure. Figure 8(a) shows the top ranked 3D models, retrieved from a sketched "human" query image. Several incorrectly retrieved 3D models from a "human" query image, like "bear" or "octopus" have quite a similar shape and pose to the sketched viewpoint. Figure $8(\mathrm{~b})$ visualizes the variation of the similarity of the proposed feature vector computation from 0.71 to 0.12 among 260 3D models. As shown in Figure 8(b), the similarity measure is quickly decreased when the retrieved $3 \mathrm{D}$ model is a "bear".

We can also approximately recover the view the user had in mind, when drawing the sketch query image as shown in Figure 8(c). The example query image is sketched from the front viewpoint of the target object. The closest views of the $3 \mathrm{D}$ models are also full-frontal or slightly in profile. One of the advantages of our method is, that we can also estimate the user's viewpoint of the query object.

Figure 9 shows the retrieved 3D models from different

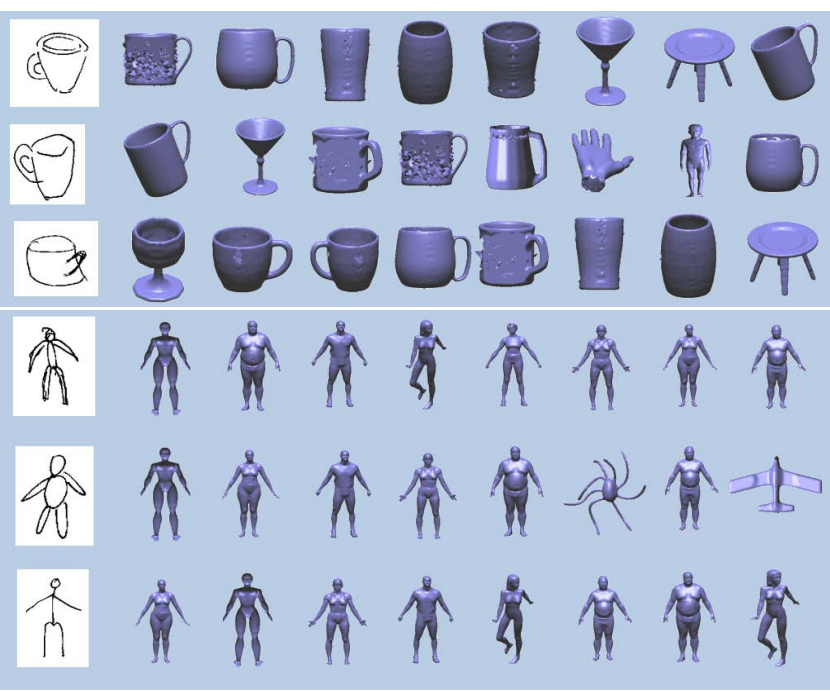

Figure 10: Comparison of retrieved 3D model from different users for "cup" and "human" respectively. The rank of the retrieved $3 \mathrm{D}$ models is dependent on the sketch's shape and viewpoint.

user-sketched query images like "chair", "cup", "bear", "ant", "hand", "human", and "sunglasses". In the top ranked 3D models as shown in Figure 9, some 3D models are incorrectly retrieved because the projected images of the $3 \mathrm{D}$ model are very similar to user's sketches or the sketched query images are very rough to retrieve the correct $3 \mathrm{D}$ models from the database.

We also tested our proposed method on various users to see how our system performs on input query images that differ from user to user. We asked users to draw the target object and searched for the closed hits in the database. Figure 10 shows the retrieved "chair" and "human" 3D models from 3 different users. As we asked them to draw the target object without specifying further information, so the drawn sketch images differ drastically from user to user.

\subsection{Comparison of Retrieval Performance}

Unfortunately, it is difficult to directly compare our proposed method to previous sketch based 3D model retrieval approaches because there are no open database of user drawn sketches to compete each other.

We therefore limit our comparison to different renderings techniques. Since we argue that suggestive contours are especially suitable for sketch-based retrieval, we conducted experiments concerning the difference of the retrieved results when rendering views using only the outer contour (silhouette) or the complete set of ridges and valleys.

To evaluate this we present the first tier precision of the human class for several, vastly different query images. Some users draw the outer shape of the target object (either with or without perspective information), others draw skeletal features of the target object.

As shown in Figure 11(a), suggestive contours yield the best retrieval result on average. It works particularly well for sketches which convey shape at least a little perspectively (user 1 and 8). Boundary contour and ridge and valley based view descriptors seem to work better for special cases. For example when users sketch skeletal features (like user 9 


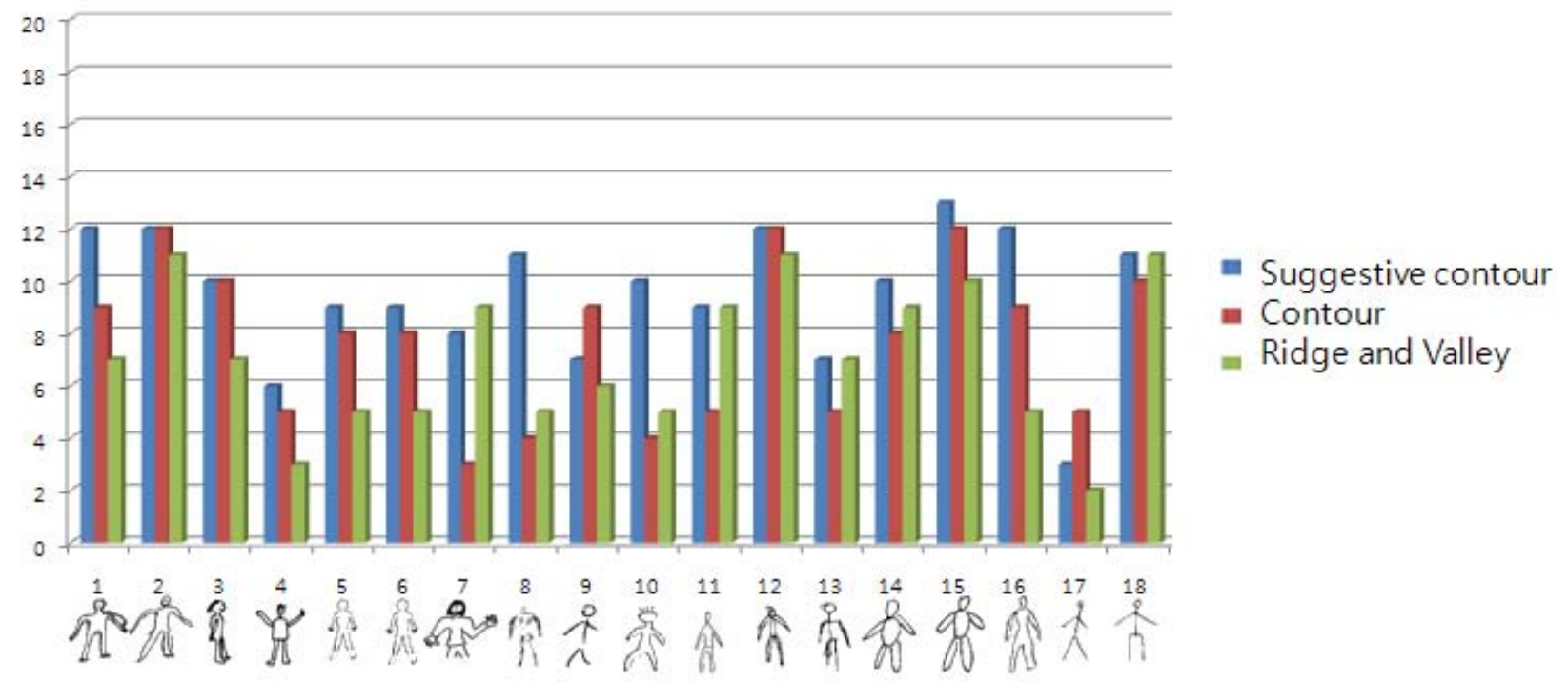

(a) First tier retrieval precision (number of correctly retrieved models out of 20 possible) for humanoid sketch-querys among several users to compare suggestive contour rendering against silhouette and ridges-and-valleys.

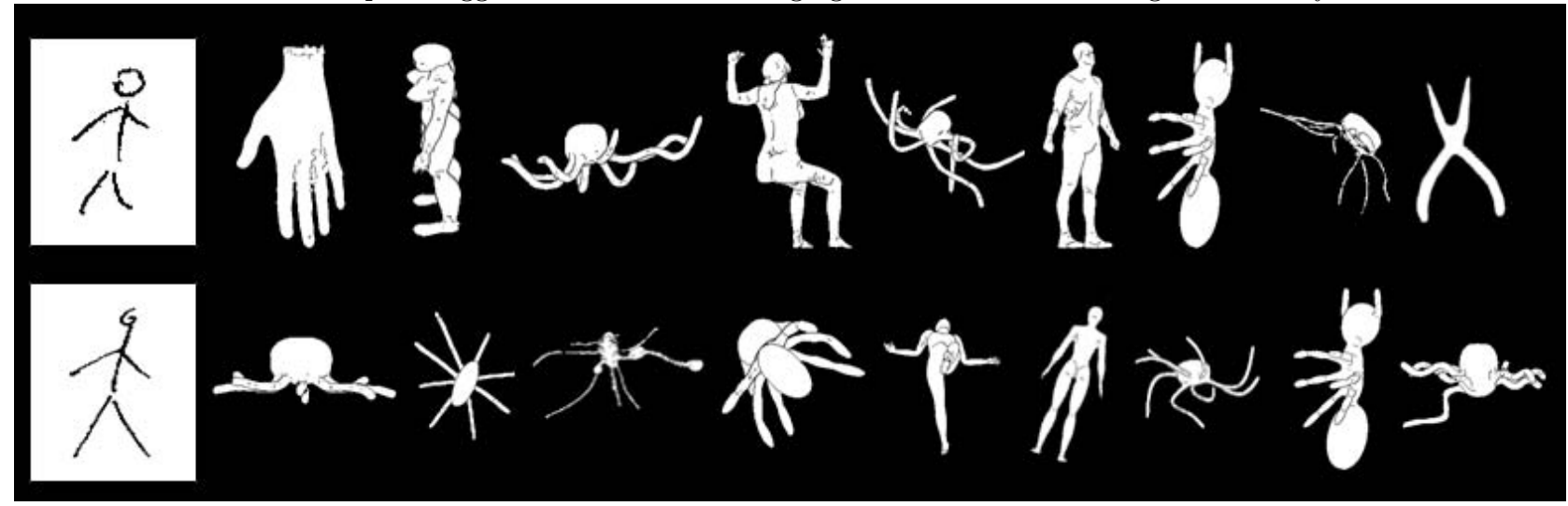

(b) The (incorrectly) retrieved models for user 9 and 17.

Figure 11: Figure 11: First tier precision (number of retrieved humanoid models out of 20) for several user-sketched query images and and a comparison of suggestive contour, contour (silhouette) and ridges and valleys.

and 17), contour seems to work rather well. Figure 11(b) shows the retrieved results user 9 and 17. As they opted to sketch skeletal features, which are not similar to the suggestive contour of a humanoid model at all, the results are rather arbitrary.

\section{DISCUSSION}

In this paper, we have presented an new approach for sketch-based 3D model retrieval using suggestive contours. To extract meaningful features from a 3D model we rendered the suggestive contour from various viewpoint. The suggestive contour was then analyzed in the space of diffusion tensor fields, and each pixel is represented as an ellipsoidal model whose direction and scale are determined by its eigenvalues and eigenvectors. The histogram of orientation is computed and the similarity between a sketch image and a 3D model can be evaluated. Our proposed method was shown to be robust against variations of shape and pose of user drawn sketches. Furthermore the suitability of suggestive contours for sketch-based retrieval was shown by comparing results against purely silhouette and ridgesand-valleys based approaches.

Based on our approach, we could also retrieve the most similar viewpoints of each 3D model as intended by the users sketch. This information might be usefully applied to project the 3D model into real-world scenes in the area of augmented reality.

\section{REFERENCES}

[1] J. W. H. Tangelder and R. C. Veltkamp A survey of content based 3D shape retrieval methods. Multimedia Tools Appl., 39(3):441-471, 2008.

[2] M. Ankerst, G. Kastenmuller, H. P. Kriegel, and T. Seidl 3D shape histogram for similarity search and classification. SSD LNCS, 1651:207-226, 1999. 
[3] R. Osada, T. Funkhouser, B. Chazelle, and D. Dobkin Matching 3D models with shape distributions. In proceeding of Shape Modeling and Applications, 154-166, 2001.

[4] M. Elad, A. Tal, and S. Ar Content based retrieval of VRML objects - an iterative and interactive approach. In proceeding of Eurographics Workshop on Multimedia, 97-108, 2001.

[5] D. Y. Chen, X. P. Tian, Y. T. Shen, and O. Ming On visual similarity based $3 \mathrm{D}$ model retrieval. In Eurographics, Computer Graphics Forum, 223-232, 2003.

[6] M. Kazhdan, T. Funkhouser, and S. Rusinkiewicz Rotation invariant spherical harmonic representation of 3D shape descriptors. In proceeding of the Symposium on Geomery Processing, 156-164, 2003.

[7] H. H. S. Ip, A. K. Y. Cheng, W. Y. F. Wong, and J. Feng. Affine-invariant sketch-based retrieval of images. In proceeding of ICCG., 2001.

[8] G. Yang, and Y. Xiao A robust similarity measure method in CBIR system. In proceeding of Congress on ISP, 2008.

[9] S. M. Yoon, and A. Kuijper Object retrieval based on user-drawn sketches. In proceeding of VISIGRAPP to be appear, 2010.

[10] D. DeCarlo, A. Finkelstein, S. Rusinkiewicz, and A. Santella Suggestive contours for conveying shape. ACM Transactions on Graphics (Proc. SIGGRAPH), 22(3):848-855, 2003.

[11] D.V. Vranic 3D model retrieval. University of Leipzig, Germany, 2004.

[12] P. Daras and A. Axenopoulos A compact multi-view descriptor for 3D object retrieval. International Workshop on Content-Based Multimedia Indexing, 115-119, 2009.

[13] L. J. Latecki, R. Lakaemper and U. Eckhardt Shape descriptors for non-rigid shapes with a single closed contour. IEEE Computer Society Conference on Computer Vision and Pattern Recognition, 1063-6919, 2000.

[14] R. Datta, J. Li, J. Z. Wang Content-based image retrieval: approaches and trends of the new age. $M I R$ '05: Proceedings of the 7th ACM SIGMM international workshop on Multimedia information retrieval, 253-262, 2005.

[15] F. Mokhtarian, S. Abbasi, and J. Kittler Efficient and robust retrieval by shape content through curvature scale space. Image Databases and Multi-Media Search, 35-42, 1996.

[16] H. Hse, and A. Richard Newton Sketched symbol recognition using zernike moments. International Conference on Pattern Recognition, 367-370, 2004.
[17] N. Dalal and B. Triggs Histograms of oriented gradients for human detection. IEEE Computer Society Conference on Computer Vision and Pattern Recognition, 886-893, 2005.

[18] T. Funkhouser, P. Min, M. Kazhdan, J.Chen, A. Halderman, and D. Dobkin A search engine for 3D models. ACM Transaction on Graphics, 22(1), 83-105, 2003.

[19] D.-Y. Chen, X.-P. Tian, Y.-T. Shen, and M. Ouhyoung On visual similarity based 3D model retrieval. Computer Graphics Forum, 22(3), 2003.

[20] D. Macrini, A. Shokoufandeh, S. Dickenson, K. Siddiqi, and S. Zucker View based 3D object recognition using shock graphs. International Conference on Pattern Recognition, 2002.

[21] C. M. Cyr, and B. Kimia 3D object recognition using shape similarity based aspect graph. International Conference on Computer Vision, 254-261, 2001.

[22] T. J. Pu, K. Lou, and K. Ramani A 2D sketch-based user interface for 3D CAD model retrieval. Computer Aided Design Application, 2(6):717-725, 2005.

[23] A. Hertzmann Introduction to 3D Non-Photorealistic Rendering: Silhouettes and Outlines. $A C M$ SIGGRAPH 99 Course Notes, 1999.

[24] Kanai, Satoshi Content-based 3D mesh model retrieval from hand-written sketch. International Journal on Interactive Design and Manufacturing, 87-98, 2008.

[25] J. Pu and K. Ramani A 3D Model Retrieval Method Using 2D Freehand Sketches. Emory University, 2005.

[26] P. J. Basser, J. Mattiello, and D. Le Bihan MR diffusion tensor spectroscopy and imaging. Biophys Journal, 1994.

[27] S. M. Yoon, and H. Graf Automatic skeleton extraction and splitting in diffusion tensor fields. IEEE International Conference on Image Processing, 2009.

[28] M. Kazhdan, B. Chazelle, D. Dobkin, and T. Funkhouser A reflective summary descriptor for 3D models. Algorithmica, 38(1):201-225, 2004.

[29] C. Zhang, and T. Chen Indexing and retrieval of 3D models aided by active learning. ACM Multimedia, 2001.

[30] C. Y. Ip, D. Lapadat, L. Sieger, W. C. Regli Using shape distributions to compare solid models. ACM Solid Modeling, 273-280, 2002.

[31] A. E. Johnson, and M. Herbert Using spin images for efficient object recognition in cluttered $3 \mathrm{D}$ scenes. IEEE PAMI, 1999.

[32] S. Kanai Content based 3D mesh model retrieval from hand-written sketch. International Journal of Interact Design Manufacture, 87-98, 2008. 